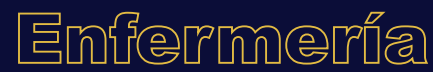

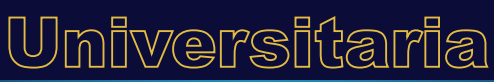

\section{Sensibilidad ética del personal de enfermería para la atención al paciente con consumo de alcohol}

\section{Ethical sensitivity among nursing personnel regarding the attention to the alcohol consuming patient}

\section{Sensibilidade ética da equipe de enfermagem para a assistência ao paciente com consumo de álcool}

\section{E. Martínez-Castilloa , M.G.E. Vázquez-Treviño ${ }^{\text {b* }}$, C.A. Carreón-Gutiérrez ${ }^{c}$, M.G. Vázquez-Salazar ${ }^{\mathrm{d}}$, L.R. De los Reyes-Nieto}

ORCID

$\begin{array}{ll}{ }^{\mathrm{a}} \underline{0000-0001-7226-3879} & { }^{\mathrm{d}} \underline{00000-0002-5696-0073} \\ { }^{\mathrm{b}} \underline{0000-0001-7848-8016} & \underline{{ }^{\mathrm{0}} 0000-0002-8395-2992}\end{array}$

c $0000-0003-2511-2220$

Universidad Autónoma de Tamaulipas, Facultad de Enfermería, Ciudad Victoria, Tamaulipas, México

Recibido: 22 septiembre 2020

Aceptado: 25 abril 2021

RESUMEN

Introducción: El consumo de bebidas alcohólicas es un problema de salud pública en el que los consumidores afectados son atendidos en los servicios de salud. El profesional de enfermería tiene un papel crucial en la atención de estos pacientes; sin embargo, los niveles de sensibilidad ética afectan los servicios brindados.

*Autor para correspondencia. Correo electrónico: gvazquez@docentes.uat.edu.mx https://doi.org/10.22201/eneo.23958421e.2021.1.994

1665-7063/@ 2021 Universidad Nacional Autónoma de México, Escuela Nacional de Enfermería y Obstetricia. Este es un artículo Open Access bajo la licencia CC BY-NC-ND (http://creativecommons.org/licenses/by-nc-nd/4.o/). 
Objetivo: Comparar el nivel de sensibilidad ética del personal de enfermería en la atención al paciente que consume alcohol, considerando las características sociodemográficas y prevalencia de consumo de alcohol.

Métodos: Estudio descriptivo, transversal y comparativo. La muestra fue de 89 profesionales de enfermería que laboran en una institución del sector público de Tamaulipas, México. Se utilizó el Cuestionario de Sensibilidad Ética y el Cuestionario de prevalencia de consumo de alcohol.

Resultados: El nivel de sensibilidad ética en el grupo de profesionales de enfermería estudiados es regular (Mediana=6.44). No se encontraron diferencias significativas por características sociodemográficas ni por prevalencia de consumo de alcohol, sólo en la carga moral entre los que consumieron alcohol en el último año ( $p<0.05)$.

Discusión: Las características sociodemográficas no influyen en el nivel de sensibilidad ética. Sólo la carga moral mostró diferencias significativas en aquellos profesionales consumidores de alcohol en el último año, lo cual implica que no están preparados para atender a pacientes con problemas de consumo de alcohol.

Conclusiones: El personal de enfermería no se encuentra preparado para la atención de pacientes con problemas de alcohol ya que los niveles de sensibilidad ética son en lo general regulares.

Palabras clave: Sensibilidad ética; personal de enfermería; consumo de bebidas alcohólicas; México.

\section{ABSTRACT}

Introduction: The consumption of alcoholic beverages is a public health problem and the related patients are attended in health services facilities. Nursing personnel have a crucial role in the attention to these patients, but the levels of ethical sensitivity which these professionals demonstrate can have impacts on the services they provide.

Objective: To compare the level of ethical sensitivity of nursing personnel regarding the attention to alcohol consuming patients, while considering social and demographic characteristics and alcohol use prevalence.

Methods: This is a descriptive, transversal, and comparative study. The sample was constituted by 89 nursing professionals working in a healthcare institution of the public sector in the state of Tamaulipas, Mexico. An Ethical Sensitivity Questionnaire and an Alcohol Consumption Prevalence Questionnaire were used.

Results: The level of ethical sensitivity in the group of professionals studied turned out to be "regular" (Median=6.44). No significant differences were found considering the social and demographic characteristics or the prevalence of alcohol consumption, except in the moral burden component among those who consumed alcohol in the last year $(p<0.05)$.

Discussion: The social and demographic characteristics do not seem to have an influence on the level of ethical sensitivity among these professionals, with the exception of those healthcare providers who used alcohol in the last year, suggesting that they might not be appropriately prepared to attend patients with problems of alcohol consumption.

Conclusions: Considering their regular levels of ethical sensitivity, these nursing professionals might not be appropriately prepared to attend patients with problems of alcohol consumption.

Keywords: Ethical sensitivity; nursing staff; alcohol drinking; Mexico. 


\section{RESUMO}

Introdução: O consumo de bebidas alcoólicas é um problema de saúde pública em que os consumidores afetados são atendidos nos serviços de saúde. O profissional de enfermagem tem um papel fundamental na assistência destes pacientes; no entanto, os níveis de sensibilidade ética afetam os serviços prestados.

Objetivo: Comparar o nível de sensibilidade ética do pessoal de enfermagem na assistência ao paciente que consome álcool, considerando as características sociodemográficas e prevalência de consumo de álcool.

Métodos: Estudo descritivo, transversal e comparativo. A amostra foi de 89 profissionais de enfermagem que laboram em uma instituição do setor público de Tamaulipas, no México. Foram utilizados o Questionário de Sensibilidade Ética e o Questionário de prevalência de consumo de álcool.

Resultados: O nível de sensibilidade ética no grupo de profissionais de enfermagem estudado é regular (Mediana=6.44). Não se encontraram diferenças significativas por características sociodemográficas nem pela prevalência de consumo de álcool, apenas na carga moral entre aqueles que consumiram álcool no último ano ( $p<0.05$ ).

Discussão: As características sociodemográficas não influenciam o nível de sensibilidade ética. Apenas a carga moral apresentou diferenças significativas naqueles profissionais consumidores de álcool no último ano, o qual envolve que não estão preparados para atender pacientes com problemas de consumo de álcool.

Conclusões: O pessoal de enfermagem não está preparado para a assistência de pacientes com problemas de álcool, dado que os níveis de sensibilidade ética geralmente são regulares. Palavras chave: Sensibilidade ética; recursos humanos de enfermagem; consumo de bebidas alcoólicas; México.

\section{INTRODUCCIÓN}

Uno de los problemas de mayor impacto en salud púbica se relaciona con el trastorno por ingesta de alcohol, ya que 5.1\% de la carga mundial de morbilidad y de lesiones se le atribuye a este factor. Igual de alarmante es la situación de la defunción temprana, donde $13.5 \%$ de las muertes que se presentan entre las edades de 20 a 39 años son atribuibles al abuso de alcohol. Aunado a lo anterior, el consumo nocivo de bebidas alcohólicas genera problemas de salud mental, complicaciones con las enfermedades no transmisibles, incremento en traumatismos, así como de pérdidas sociales y económicas importantes, tanto para la persona que tiene esta enfermedad, como la familia del mismo.

En México el alcoholismo es considerado como el responsable directo del aumento de la carga de la enfermedad ${ }^{2}$. De acuerdo con Pinheiro-Gawrysewsky y Monteiro3, México se encontró entre los primeros cinco países con mayor tasa de mortalidad por consumo de alcohol en América Latina. El porcentaje de dependencia al alcohol a nivel nacional en 2017 fue de $6.2 \%$, la prevalencia de alguna vez haber consumido bebidas alcohólicas es mayor en los hombres con 80.1\%, y en las mujeres alcanza $62.6 \% 4$.

En cuanto a los servicios de salud brindados a la población consumidora de alcohol en México, se identificó que, de los 13405 eventos registrados en urgencias hospitalarias en 2016, sólo 3.9\% de los pacientes se encontraban bajo el efecto de alguna droga, de ellos, $67.8 \%$ fue por consumo de alcohol. El servicio médico forense reportó que, de los 11356 casos registrados en 2016, 8.6\% presentó 
evidencia del uso de una sustancia, de la cual el alcohol es la que registró mayor frecuencia (66.6\%). En Tamaulipas se reporta una prevalencia de consumo diario de bebidas alcohólicas del 2.4\%, mientras que el consumo consuetudinario es del 10.5\%4.

Al existir una asociación entre el consumo de alcohol con los problemas de salud, las personas con esta enfermedad acuden y son atendidas frecuentemente en servicios hospitalarios debido a complicaciones que se presentan ${ }^{4.5}$. La profesión de enfermería tiene un papel crucial en la atención y el cuidado a la salud, incluyendo los pacientes con problemas de consumo de sustancias. No obstante, las actitudes que presenta el personal de enfermería hacia este grupo de pacientes pueden verse modificadas o influenciadas por sentimientos personales, experiencias previas, creencias, valores, empatía y conocimientos ${ }^{6,7}$.

La percepción que la enfermera tiene del paciente es el principal determinante de la calidad de cuidados prestados ${ }^{6}$. En el caso del paciente con trastorno por ingesta de alcohol no es la excepción; sin embargo, en la mayor parte de los casos la atención brindada suele ser problemática ${ }^{8,9}$. Se ha documentado el efecto de las actitudes negativas que presentan los profesionales de enfermería hacia el paciente con abuso de bebidas alcohólicas dando como resultado atención a la salud inapropiada, con la cual se generan cuidados que pueden perjudicar el curso del tratamiento $0^{6,9-11}$.

El cuidado constituye la base de la actividad de la enfermera, éste incluye no sólo comportamientos científicos y técnicos, sino que además considera acciones de alivio al sufrimiento humano, mantenimiento de la dignidad, manejo adecuado en situaciones de crisis, empatía y experiencia de vivir y morir ${ }^{12-14}$. En el cuidado de la salud se utiliza el concepto de sensibilidad ética para describir el componente de la toma de decisiones en el cuidado profesional, el cual corresponde a la sensibilidad moral, percepción moral, ética, intuición y moral ${ }^{15}$.

La sensibilidad ética contempla la orientación interpersonal (relación entre el profesional y la persona a la que se cuida), estructuración del significado moral (evaluar decisiones y acciones), la autonomía (protegiendo el cuidado del paciente de auto-daño), benevolencia (estar motivado para actuar en el mejor interés del paciente), la identificación de sentimientos subyacentes como la intuición y la percepción de situaciones sobre el cuidado del paciente que exige al profesional tomar acción, y la confianza en el conocimiento médico y de enfermería ${ }^{16}$.

Los trabajos de Lützén et al. ${ }^{17-19}$ se enfocan en evaluar la sensibilidad ética en la práctica de los profesionales de la salud, la cual puede verse afectada por diversos factores, entre ellos los demográficos o características del personal. De hecho, desde su trabajo de 2006 se propone un instrumento que permite valorar la sensibilidad ética a partir de tres dimensiones: el sentido de carga moral, fortaleza moral y responsabilidad moral ${ }^{17}$. La carga moral se considera una dimensión «negativa», representa algo a lo que el personal de salud no está "preparado». La fortaleza moral es expresada como el «valor de actuar» y la capacidad para justificar las acciones realizadas en beneficio de los demás ${ }^{17-20}$. Por otro lado, la responsabilidad moral está asociada a la obligación moral de trabajar conforme a las normas y reglamentaciones ${ }^{20}$.

La deshumanización en la atención de salud coloca en evidencia la ausencia de comportamientos bioéticos en los profesionales de enfermería, reflejada en la falta de respeto y agresión a la dignidad de los pacientes. Además, los profesionales de enfermería enfrentan actitudes y estigmas hacia los pacientes con problemas de consumo de alcohol ya que se cree que son personas desagradables, difíciles de atender, peligrosos, de voluntad débil e inmorales. Esto se asocia al juicio de cómo merecen ser tratados y todas estas creencias pueden moldear e influir la relación enfermera-paciente ${ }^{16}$. 
Por tal motivo, es importante evaluar las actitudes y sensibilidad del profesional de enfermería al proveer el cuidado. El presente trabajo tiene como finalidad comparar el nivel de sensibilidad ética del personal de enfermería en la atención al paciente con trastorno del consumo de bebidas alcohólicas, considerando las características sociodemográficas y hábitos de consumo de alcohol, en una institución hospitalaria del sector público de Ciudad Victoria, Tamaulipas, México.

\section{METODOLOGÍA}

Se trata de un estudio descriptivo, transversal y correlacional. Se realizó durante los meses de septiembre a octubre del 2019. La población diana estuvo conformada por profesionales de enfermería que laboran en una institución hospitalaria del sector público de Ciudad Victoria, Tamaulipas, México. Los criterios de inclusión fueron profesionales de enfermería con contrato definitivo, de los diferentes turnos laborales, con nivel educativo de licenciatura en enfermería o superior, con al menos un año de antigüedad laboral en la institución y que aceptaran participar a través del consentimiento informado. Los criterios de exclusión comprenden profesionales de enfermería eventuales, que no han cumplido el año de antigüedad laboral en la institución, que laboren en áreas administrativas o con licencia de trabajo y aquellos que decidieron no participar.

La población de investigación está conformada por el total de la plantilla de personal de enfermería constituida por 121 profesionales. A partir de establecer los criterios de inclusión y exclusión se obtuvo una muestra final de 89 profesionales de enfermería. Se realizó muestreo por conveniencia.

Para la recolección de datos se utilizó una encuesta autoadministrada, la cual estaba integrada por una cédula de datos sociodemográficos que incluía las variables (edad, sexo, estado civil, religión, escolaridad); así como la variable prevalencia de consumo de alcohol. Otro instrumento empleado fue el Cuestionario de Sensibilidad Ética (CSE) de Lützén et al. ${ }^{17}$, validado previamente en población mexicana por Almaraz et al. ${ }^{20}$. En su totalidad la aplicación de la encuesta tuvo una duración aproximada de 30 minutos, la cual fue respondida por el personal de enfermería durante el turno laboral.

La cédula de datos sociodemográficos se diseñó con preguntas cerradas para las variables sexo (hombre, mujer), estado civil (soltero, casado, divorciado, separado, unión libre, viudo), grado académico (practicante, licenciatura, especialidad, maestría). Para el caso de las variables de edad y religión, las preguntas fueron abiertas, las cuales posteriormente se categorizaron para el análisis estadístico. La variable prevalencia de consumo de alcohol está conformada por cuatro ítems, con respuestas dicotómicas de Sí/No. Las preguntas estaban orientadas a conocer si las personas consumieron alcohol alguna vez en la vida, en el último año, en el último mes, y/o en la última semana.

El CSE reporta un alfa de Cronbach de $0.7^{20}$ en profesionales de enfermería mexicanos y consta de 9 ítems con seis posibles respuestas en escala tipo Likert desde 1 (completamente en desacuerdo) a 6 (completamente de acuerdo). A mayor puntaje, mayor será la sensibilidad ética. El instrumento permite valorar tres componentes de la sensibilidad ética: sentido de la carga moral (ítems 4, 6, 7 y

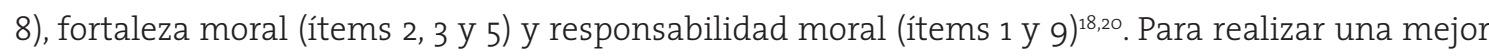
interpretación las puntuaciones obtenidas se transformaron a una escala base 10, donde los valores cercanos a 10 representan mayor sensibilidad ética. Para este estudio, el CSE reportó un alfa de Cronbach de 0.77 que es una consistencia interna aceptable.

Los datos recolectados fueron analizados estadísticamente con el paquete Statistics Program for Social Science (SPSS) versión 20. Para la descripción de las características del personal de enfermería participante se empleó frecuencias y porcentajes. Para el análisis de los valores de sensibilidad ética y sus dimensiones se usó estadística descriptiva, reportando los datos que mejor explicaran 
la naturaleza de los valores obtenidos. El análisis correlacional se obtuvo a través de la prueba de medianas, se consideró que había diferencias significativas entre grupos cuando presentaban un valor de $\mathrm{p} \leq 0.05$.

La investigación y la carta de consentimiento informado contó con la aprobación del Comité de Ética de la Facultad de Enfermería Victoria de la Universidad Autónoma de Tamaulipas (Oficio ○02/2018), así como la autorización de los directivos de la institución hospitalaria donde se realizó el estudio. El estudio se apegó a los lineamientos del Reglamento de la Ley General de Salud en Materia de Investigación para la Salud y a la Declaración de Helsinki.

\section{RESULTADOS}

La caracterización sociodemográfica de los profesionales de enfermería se muestra en la tabla 1. 86.5\% corresponde al sexo femenino; en cuanto a la edad, se identificó que el personal masculino es más joven con una mediana de 27 años, mientras que la edad promedio de la muestra femenina es de 43 años. 59.6\% del personal encuestado se encuentran casados; $82.0 \%$ profesa la religión católica y $52.8 \%$ posee grado de licenciatura. Cabe señalar que 38.2\% de los profesionales de enfermería indicaron tener grado de especialidad.

En cuanto al consumo de alcohol, al menos $66.2 \%$ de los participantes indicaron haber consumido bebidas alcohólicas alguna vez en su vida; $39.3 \%$ reportó el consumo de alcohol en el último año; $28.8 \%$ de los profesionales señaló haber consumido alcohol en el último mes y 17.9\% consumió alcohol en la última semana. En todos los casos, la mayor prevalencia fue reportada en los hombres (Tabla 1).

\begin{tabular}{|c|c|c|c|}
\hline \multirow[t]{2}{*}{ Variable } & \multicolumn{2}{|c|}{ Sexo } & \multirow[t]{2}{*}{ General } \\
\hline & Hombre & Mujer & \\
\hline Distribución, n (\%) & $12(13.5)$ & $77(86.5)$ & $89(100.0)$ \\
\hline $\begin{array}{l}\text { Edad, años } \\
\text { Mediana (Q1-Q3) }\end{array}$ & $27.0(25.0-29.0)$ & $43.0(36.0-52.0)$ & $40.0(29.0-50.0)$ \\
\hline \multicolumn{4}{|l|}{ Edad, años n (\%) } \\
\hline De 18 a 25 & $5(41.7)$ & $3(3.9)$ & $8(9.0)$ \\
\hline De 26 a 33 & $6(50.0)$ & 15 (19.5) & 21 (23.6) \\
\hline De 34 a 40 & $1(8.3)$ & 15 (19.5) & $16(18.0)$ \\
\hline De 41 a 50 & --- & $22(28.5)$ & $22(24.7)$ \\
\hline De 51 a 60 & --- & $21(27.3)$ & $21(23.6)$ \\
\hline De 61 o más & --- & $1(1.3)$ & $1(1.1)$ \\
\hline \multicolumn{4}{|l|}{ Estado civil, n (\%) } \\
\hline Soltero & $7(58.3)$ & $11(14.3)$ & $18(20.2)$ \\
\hline Casado & $5(41.7)$ & $48(62.3)$ & $53(59.5)$ \\
\hline Divorciado & --- & $3(3.9)$ & $3(3.4)$ \\
\hline Separado & --- & $3(3.9)$ & $3(3.4)$ \\
\hline Unión libre & --- & $11(14.3)$ & $11(12.4)$ \\
\hline Viudo & --- & $1(1.3)$ & $1(1.1)$ \\
\hline
\end{tabular}




\begin{tabular}{lccc}
\hline Religión, $\mathrm{n}(\%)$ & $9(75.0)$ & $64(83.1)$ & $73(82.0)$ \\
\hline Católica & $2(16.7)$ & $10(13.0)$ & $12(13.5)$ \\
\hline Cristiana & --- & $1(1.3)$ & $1(1.1)$ \\
\hline Otra & $1(8.3)$ & $2(2.6)$ & $3(3.4)$ \\
\hline No contestó & & & $1(1.1)$ \\
\hline Grado académico, $\mathrm{n}(\%)$ & $1(8.3)$ & --- & $47(52.8)$ \\
\hline Practicante & $6(50.0)$ & $31(53.2)$ & $34(38.2)$ \\
\hline Licenciatura & $4(33.4)$ & $3(39.0)$ & $3(4.5)$ \\
\hline Especialidad & $1(8.3)$ & $3(3.9)$ & $3.4)$ \\
\hline Maestría & --- & & $59(66.2)$ \\
\hline No contestó & $9(75.0)$ & $50(64.9)$ & $35(39.3)$ \\
\hline Prevalencia de consumo de alcohol, $\mathrm{n}(\%) *$ & $26(33.7)$ & $25(28.1)$ \\
\hline Alguna vez en la vida & $9(75.0)$ & $16(20.7)$ & $16(17.9)$ \\
\hline En el último año & $9(75.0)$ & $12(15.5)$ & \\
\hline En el último mes & $4(33.3)$ & & \\
\hline En la última semana &
\end{tabular}

${ }^{*}$ Se reportan los casos afirmativos

Los niveles de sensibilidad ética, tanto de forma general como por variables sociodemográficas y de consumo de alcohol se pueden observar en la tabla 2. De forma general, el nivel de sensibilidad ética que reporta el grupo de estudio es de una mediana=6.44, interpretado como regular. Por componentes, se observa el mismo tipo de comportamiento, siendo la responsabilidad moral la que mejor puntuación presenta en el grupo de profesionales estudiados con una mediana=7.00.

La dimensión sensibilidad ética se comportó de manera diferente por sexo, el personal masculino obtuvo una mediana de 6.56, ligeramente mayor en contraste con los niveles que correspondieron a las enfermeras (mediana $=6.44$ ). Los mejores resultados de sensibilidad ética se observan en los grupos de 51 a 60 años con una mediana de 7.56. El personal cuyo estado civil es separado registró una mediana de 8.44 y el personal que profesa el cristianismo registró una mediana de 6.67 (Tabla 2).

Tabla 2. Sensibilidad ética en personal de enfermeria por características sociodemográficas

Variables de agrupación
Sensibilidad ética

Mediana (Q1-Q3)

\begin{tabular}{lcccc}
\cline { 2 - 4 } & Carga Moral & Fortaleza Moral & $\begin{array}{c}\text { Responsabilidad } \\
\text { Moral }\end{array}$ & Nivel Global \\
\hline $\begin{array}{l}\text { General } \\
\text { Sexo }\end{array}$ & $6.00(5.00-7.00)$ & $6.11(5.00-7.22)$ & $7.00(5.00-9.00)$ & $6.44(5.56-7.56)$ \\
Hombre & $6.00(5.00-7.75)$ & $6.11(5.00-7.22)$ & $7.50(6.00-9.50)$ & $6.56(6.00-8.11)$ \\
\hline Mujer & $6.00(4.50-7.00)$ & $6.11(5.00-7.22)$ & $7.00(5.00-9.00)$ & $6.44(5.33-7.56)$
\end{tabular}




\begin{tabular}{|c|c|c|c|c|}
\hline \multicolumn{5}{|l|}{ Edad } \\
\hline De 18 a 25 años & $5.00(4.75-7.00)$ & $6.11(5.56-7.22)$ & $7.00(4.00-8.00)$ & $6.44(5.89-7.11)$ \\
\hline De 26 a 33 años & $6.00(5.00-7.50)$ & $5.00(5.00-7.22)$ & $8.00(6.00-9.00)$ & $6.44(5.56-8.00)$ \\
\hline De 34 a 40 años & $5.50(4.25-7.00)$ & $5.00(3.61-7.22)$ & $6.50(4.00-9.00)$ & $6.22(4.33-7.56)$ \\
\hline De 41 a 50 años & $6.00(4.50-7.00)$ & $5.83(4.44-7.22)$ & $6.00(5.00-8.00)$ & $6.11(5.33-6.67)$ \\
\hline De 51 a 60 años & $6.50(5.00-7.50)$ & $6.67(5.11-7.78)$ & $8.00(6.00-10.00)$ & $7.56(6.00-8.22)$ \\
\hline De 61 o más años & $4.50(4.50-4.50)$ & $5.56(5.56-7.00)$ & $7.00(7.00-7.00)$ & $5.78(5.78-5.78)$ \\
\hline \multicolumn{5}{|l|}{ Estado civil } \\
\hline Soltero & $6.00(5.00-7.50)$ & $6.11(5.00-7.22)$ & $7.00(6.00-8.00)$ & $6.44(6.00-8.22)$ \\
\hline Casado & $6.00(5.00-7.00)$ & $6.11(4.44-7.22)$ & $7.00(5.00-9.00)$ & $6.44(5.33-7.56)$ \\
\hline Divorciado & $4.00(4.00-6.50)$ & $5.00(3.89-6.67)$ & $7.00(4.00-8.00)$ & $6.00(4.67-6.22)$ \\
\hline Separado & $7.50(4.50-8.50)$ & $7.22(6.11-8.33)$ & $10.00(9.00-10.00)$ & $8.44(6.44-9.33)$ \\
\hline Unión libre & $6.00(3.50-8.00)$ & $5.56(5.00-7.22)$ & $6.00(3.00-9.00)$ & $6.22(4.89-8.00)$ \\
\hline Viudo & $5.50(5.50-5.50)$ & $6.11(6.11-6.11)$ & $8.00(8.00-8.00)$ & $6.67(6.67-6.67)$ \\
\hline \multicolumn{5}{|l|}{ Religión } \\
\hline Católica & $6.00(5.00-7.00)$ & $6.11(5.00-7.11)$ & $7.00(6.00-9.00)$ & $6.44(5.56-7.56)$ \\
\hline Cristiana & $5.00(4.75-6.75)$ & $5.83(4.72-7.78)$ & $5.50(5.00-9.00)$ & $6.67(5.00-7.67)$ \\
\hline Otra & $4.00(4.00-4.00)$ & $3.33(3.33-3.33)$ & $4.00(4.00-4.44)$ & $4.00(4.00-4.00)$ \\
\hline \multicolumn{5}{|l|}{ Grado académico } \\
\hline Practicante & $6.00(6.00-6.00)$ & $5.00(5.00-5.00)$ & $6.00(6.00-6.00)$ & $6.00(6.00-6.00)$ \\
\hline Licenciatura & $5.50(4.50-6.50)$ & $6.11(5.00-7.22)$ & $7.00(6.00-9.00)$ & $6.44(5.56-7.66)$ \\
\hline Especialidad & $6.50(5.00-7.50)$ & $6.11(4.44-7.22)$ & $7.50(5.00-9.00)$ & $6.67(5.56-7.78)$ \\
\hline Maestría & $6.00(5.00-6.70)$ & $6.67(5.56-7.22)$ & $3.50(3.00-7.00)$ & $6.00(5.22-7.33)$ \\
\hline \multicolumn{5}{|c|}{ Consumo de alcohol* } \\
\hline $\begin{array}{l}\text { Alguna vez en la } \\
\text { vida }\end{array}$ & $6.00(4.50-7.50)$ & $6.11(5.00-7.22)$ & $7.00(5.00-8.00)$ & $6.44(5.33-7.50)$ \\
\hline En el último año & $6.00(5.00-8.00)$ & $6.11(5.00-7.22)$ & $8.00(6.00-9.00)$ & $6.44(6.00-8.00)$ \\
\hline En el último mes & $6.00(5.00-7.50)$ & $6.11(5.56-7.22)$ & $8.00(6.00-9.00)$ & $6.67(6.00-8.00)$ \\
\hline $\begin{array}{l}\text { En la última } \\
\text { semana }\end{array}$ & $6.00(5.00-6.50)$ & $6.11(5.00-7.22)$ & $7.50(5.00-8.00)$ & $6.22(5.89-7.44)$ \\
\hline
\end{tabular}
están definidos por el sexo, estado civil, edad, religión, grado de escolaridad y el consumo de alcohol. No se encontró evidencia suficiente para demostrar diferencias significativas en los valores de sensibilidad ética y sus componentes entre los elementos de cada variable demográfica. 


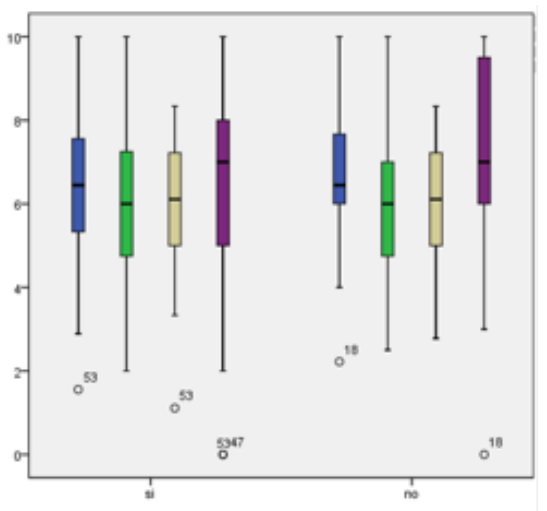

Alguna vez

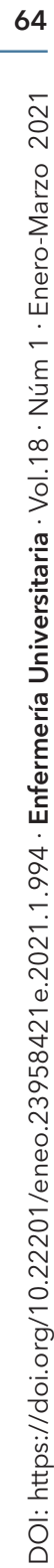

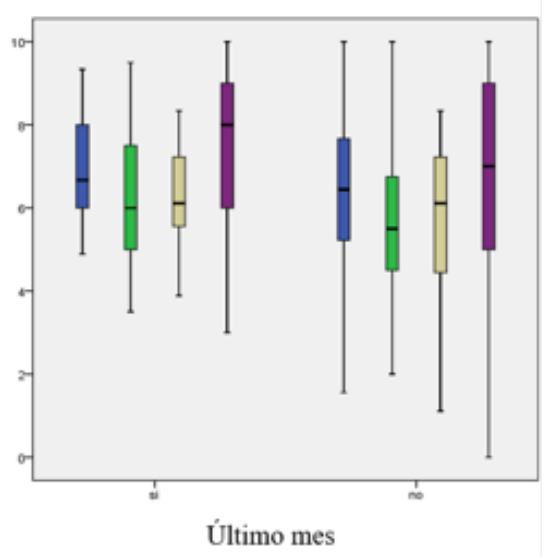

† Prueba de medianas

* Significancia al 0.05

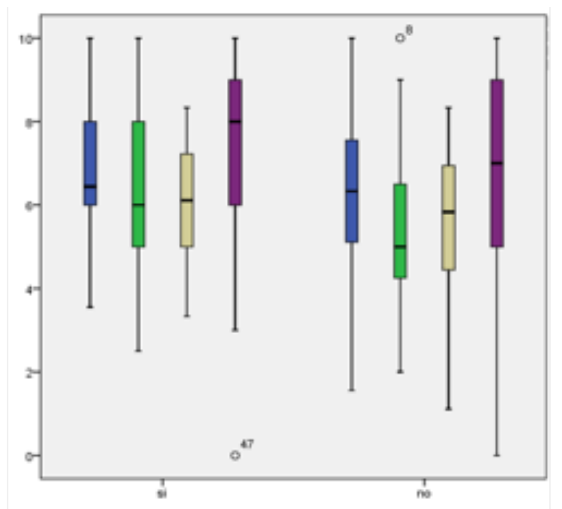

Último año

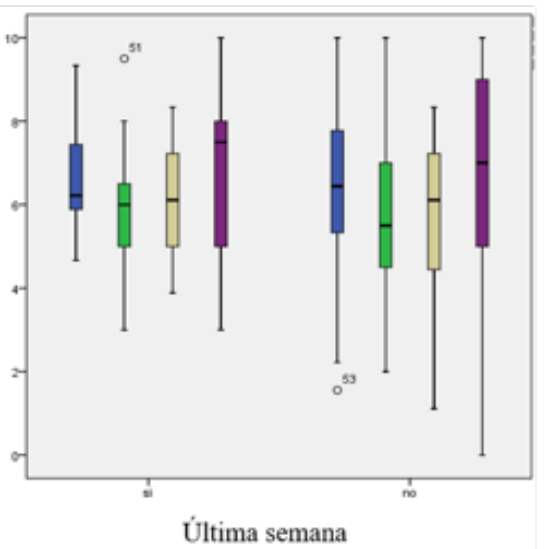

Ultima semana
Nivel de sensibilidad ética

Carga Moral

$\square$ Fortaleza Moral

Responsabilidad Moral

Figura 1. Comportamiento del índice de sensibilidad ética según el periodo de consumo

Tabla 3. Comparación del indice sensibilidad ética y sus componentes por caracterización sociodemográfica

\begin{tabular}{lcccc} 
Variable de agrupación † & $\begin{array}{c}\text { Índice de } \\
\text { sensibilidad ética }\end{array}$ & $\begin{array}{c}\text { Carga } \\
\text { Moral }\end{array}$ & $\begin{array}{c}\text { Fortaleza } \\
\text { Moral }\end{array}$ & $\begin{array}{c}\text { Responsabilidad } \\
\text { moral }\end{array}$ \\
\hline Sexo & 0.813 & 0.823 & 0.889 & 0.947 \\
\hline Estado civil & 0.489 & 0.875 & 0.868 & 0.364 \\
\hline Edad & 0.152 & 0.704 & 0.245 & 0.245 \\
\hline Religión & 0.396 & 0.591 & 0.706 & 0.422 \\
\hline Grado máximo de estudios & 0.380 & 0.195 & 0.787 & 0.614 \\
\hline Consumo de alcohol & & & & 0.728 \\
\hline Alguna vez & 0.678 & 0.475 & 0.695 & 0.207 \\
\hline En el último año & 0.783 & $0.047 *$ & 0.251 & 0.933 \\
\hline En el último mes & 0.672 & 0.250 & 0.910 & 0.970 \\
\hline En la última semana & 0.419 & 0.778 & 0.894 & \\
\hline
\end{tabular}




\section{DISCUSIÓN}

Las características demográficas del personal de enfermería encuestado indican el predominio de las mujeres, aspecto que no es nuevo gracias a la naturaleza y dinámica propia de la profesión ${ }^{21}$. En cuanto al consumo de bebidas alcohólicas, la prevalencia es mayor en los hombres en comparación con las mujeres, comportamiento similar a los reportados por la ENCODAT 2016-20174. Aunque, en contraste con los resultados mostrados por Almaraz et al. ${ }^{22}$ y Hernández et al. ${ }^{23}$, los datos que se reportan en este estudio son valores más bajos.

El nivel de sensibilidad ética identificado en los profesionales de enfermería se considera regular, resultado que se asemeja a lo que Hernández et al. ${ }^{23}$, Almaraz et al. ${ }^{20}$ y Alonso et al. ${ }^{24}$ reportaron, cuyos datos son preocupantes. El tener un nivel de sensibilidad ética bajo o con un nivel regular implica que el profesional de enfermería tiene problemas en la capacidad para realizar una adecuada toma de decisiones en el cuidado de las personas con problemas de adicción, y por ende, la calidad del servicio de enfermería se verá afectado.

La sensibilidad ética puede ser expresada de diferentes maneras en los profesionales de enfermería, incrementando el nivel de complejidad para su estudio, considerando los factores y contextos en los que se desarrolla ${ }^{18,19}$. Tanto Lützén et $a .^{18}$, Tuvesson y Lützén ${ }^{19}$ y Kulju et al.$^{25}$ determinaron que existen diferencias significativas entre el sexo y la edad con los valores de sensibilidad ética. Nuestros resultados no encontraron evidencia de una asociación entre dichas variables. Este comportamiento ya lo habían reportado previamente Alonso y Alonso ${ }^{26}$, Alonso-Castillo et al. ${ }^{24}$ y Almaraz et al..$^{20,22}$.

Con respecto a la variable sexo, Almaraz et al..$^{20}$ reportaron que en la fortaleza moral los hombres presentaron valores altos en comparación con las mujeres, mientras que Tuvesson y Lützén ${ }^{19}$ lo identificaron en las mujeres de manera general.

A pesar de que nuestro estudio determinó que no son significativas las diferencias por sexo en las dimensiones de sensibilidad ética, se observa que los valores son mayores en hombres en contraste con las mujeres, coincidiendo con los resultados de Almaraz et al. ${ }^{20}$. De igual forma, se coincide con la opinión de estos autores, quienes explican que parte de este comportamiento se puede asociar al contexto cultural y al ambiente en donde se desenvuelven los participantes del estudio.

En cuanto al nivel académico, Alonso et al. ${ }^{24,26}$ reportaron que había diferencias significativas en el nivel de sensibilidad ética, donde a mayor nivel académico, mayor es el nivel de sensibilidad éti$\mathrm{ca}^{26}$. Sin embargo, no se encontraron evidencias suficientes para diferenciar la puntuación por grado académico, aunque sí fue notorio que quienes presentaron un nivel más alto de sensibilidad ética eran los profesionales de enfermería con grado académico de especialidad.

Sobre el análisis entre los hábitos de consumo de alcohol con el nivel de sensibilidad ética, mientras Almaraz et al. ${ }^{20}$ no encontraron relación alguna entre el consumo de alcohol y el nivel de sensibilidad ética, Hernández et al. ${ }^{23}$ identificaron una relación positiva entre ambas; es decir, a mayor consumo de bebidas alcohólicas, mayor será el nivel de sensibilidad ética. Los resultados del presente estudio se asemejan más a los resultados de Almaraz et al. ${ }^{20}$ ya que no se encontró evidencia suficiente para demostrar una relación entre el consumo de alcohol y el nivel de sensibilidad ética.

A pesar de que estas dos variables no tienen relación de manera general, se identifica que existen diferencias significativas con la dimensión de carga moral en aquellas personas que consumieron bebidas alcohólicas en el último año. Es importante recordar que la carga moral es considerada como la dimensión negativa, puesto que representa algo a lo que el personal no está preparado ${ }^{18,20,22}$. En otras palabras, aquel profesional de enfermería que había consumido bebidas alcohólicas muestra niveles más bajos de carga moral que en aquellos que no lo hicieron, posiblemente porque no se habían enfrentado a una situación previa o por situaciones personales. 
En primera instancia, los consumidores de alcohol son pacientes dificiles y muchas veces no deseables ya que implican problemas físicos, mentales y espirituales ${ }^{5,11}$. Por otro lado, también se reconoce que el personal de enfermería no tiene la capacitación adecuada para la atención de este tipo de pacientes y, por ende, no están preparados adecuadamente para ofrecer cuidados a este grupo de personas $^{5,8,11}$. Es probable que estas situaciones sean el motivo del resultado de los niveles de carga moral identificados en el presente trabajo.

Van Boekel et al.10 encontraron en su revisión sistemática que existen actitudes negativas por parte del personal de salud hacia la atención de pacientes con problemas de consumo de sustancias. De acuerdo con este estudio, existe una estigmatización hacia este grupo de pacientes, la cual muchas veces es generada por las creencias y percepciones propias de los profesionales de la salud. En este punto es importante aclarar que la estigmatización no inicia en el propio profesional de salud, sino por el contexto que lo rodea, donde dichas creencias y percepciones de la sociedad las hacen propias. Por lo tanto, esta estigmatización que el personal adopta o interioriza como propia influye en la forma en la cual deben de actuar, modificando sus acciones en cuanto a sensibilidad ética se refiere.

Por otra parte, Ford et al. ${ }^{27}$ indicaron que el profesional de enfermería no tiene la suficiente formación para poder atender adecuadamente a pacientes con problemas de consumo de sustancias, siendo necesaria una capacitación, pero esto se debiera de resolver desde la formación profesional.

\section{CONCLUSIÓN}

A pesar de que no se encontraron diferencias significativas en la comparación de los valores de sensibilidad ética agrupados por nivel de consumo y caracterización sociodemográfica, debemos de tomar en cuenta los hallazgos realizados. Los hombres muestran mejores niveles de sensibilidad ética en comparación con las mujeres, implicando que muestran hasta cierto punto una mayor empatía hacia los pacientes que presentan problemas de consumo de alcohol.

Lo anterior puede ser explicado también por los hábitos de consumo de alcohol, donde el nivel de carga moral es el único afectado por esta variable. Si bien es cierto que el personal de enfermería no se encuentra preparado tanto física como mentalmente para atender a pacientes con problemas de consumo de alcohol, aquellos que han consumido bebidas alcohólicas poseen mejores niveles en esta dimensión. Aunado a esto, la falta de preparación para brindar atención a personas con problemas de alcohol es un problema que repercute directamente en la calidad de los servicios brindados por los profesionales de enfermería, ya que los niveles de sensibilidad ética son en lo general regulares.

Una de las limitaciones del presente estudio es el no haber medido la cantidad consumida ni el nivel de consumo de alcohol en los profesionales de enfermería. Por ello se hace la sugerencia que en futuros estudios se contemplen estas variables con la finalidad de describir mejor el comportamiento de consumo de alcohol. Asimismo, se recomienda realizar capacitaciones orientadas a mejorar tanto el nivel de sensibilidad ética como el nivel de resiliencia del personal de enfermería para enfrentar, de forma adecuada, situaciones que impliquen brindar atención a pacientes con problemas de consumo de sustancias. Se requiere reforzar la capacidad y competencia para detectar de manera oportuna y en etapas tempranas a pacientes consumidores de sustancias psicoactivas.

\section{RESPONSABILIDADES ÉTICAS}

Protección de personas y animales. Los autores declaran que para el desarrollo del presente estudio no se realizaron experimentos en seres humanos ni en animales. 
Confidencialidad. En este estudio no aparecen datos confidenciales de los participantes. Todos los participantes otorgaron previamente su consentimiento durante la realización del estudio. También se respetó en todo momento la confidencialidad de los datos obtenidos ya que los cuestionarios fueron anónimos.

Conflicto de intereses. Los autores declaran no tener conflicto de intereses.

Financiamiento. Ninguno.

\section{REFERENCIAS}

1. Organización Mundial de la Salud (OMS). Alcohol. Datos y cifras. Ginebra: OMS; 2018. https://bit.ly/3fHoUOLh

2. Lozano R, Gómez-Dantés H, Garrido-Latorre F, Jiménez-Corona A, Campuzano-Rincón JC, Franco-Marina F, et al. La carga de enfermedad, lesiones, factores de riesgo y desafíos para el sistema de salud en México. Salud Publica Mex. 2013; 55(6): 580-94.

http://dx.doi.org/10.21149/spm.v55i6.7304

3. Pinheiro-Gawryszewski V, Monteiro MG. Mortality from diseases, conditions and injuries where alcohol is a necessary cause in the Americas, 2007-09. Addiction. 2014; 109(4): 570-77. https://doi.org/10.1111/add.12418

4. Instituto Nacional de Psiquiatría Ramón de la Fuente Muñiz (INPRFM)/ Instituto Nacional de Salud Pública (INSP)/ Comisión Nacional Contra las Adicciones (CONADIC)/ Secretaría de Salud (SSA). Encuesta Nacional de Consumo de Drogas, Alcohol y Tabaco 2016-2017. Reporte de Alcohol. México: INPRFM; 2017.

5. Bettancourt-Ortega L, Arena-Ventura C. Estoy sola: la experiencia de las enfermeras en el cuidado del usuario de alcohol y drogas. Rev Esc Enferm USP. 2013; 47(6): 1381-8.

http://dx.doi.org/10.1590/So080-623420130000600019

6. Crothers CE, Dorrian J. Determinants of nurses' attitudes toward the care of patients with alcohol problems. ISRN Nurs. 2011: 1-12. http://dx.doi.org/10.5402/2011/821514

7. Molina-Mula J, Hernández-Sánchez D, Sanz-Álvarez E, Clar-Aragón F, Grupo de investigación Balear de enfermería en drogodependencias. Impacto de las actitudes de las enfermeras en la calidad de los cuidados en drogodependientes. Índex Enferm. 2012; 21 (4): 214-8. http://dx.doi.org/10.4321/S1132-12962012000300008

8. Vargas D, Curi-Labate R. Atitudes de enfermeiros de hospital geral frente ao uso do álcool e alcoolismo. Rev. Bras. Enferm. 2006; 59(1): 47-51. https://bit.ly/3i7eRso

9. López-Alabarce JA, Hernández-Sánchez D, Molina-Mula J, Fernández-Medina JM, Castaño-Fernández AI, Sanz-Álvarez E, et al. Percepción, conocimientos y actitudes de las enfermeras de urgencias y salud mental frente al alcoholismo y otras drogodependencias. Metas Enferm. 2014; 17(2): 22-31. https://bit.ly/34FUUAB

10. Van Boekel LC, Brouwers EPM, van Weeghel J, Garretsen HFL. Stigma among health professionals towards patients with substance use disorders and its consequences for healthcare delivery: Systematic review. Drug Alcohol Depend. 2013; 131(1-2): 23-35. https://doi.org/10.1016/j.drugalcdep.2013.02.018

11. Sánchez-Solís A, de San Jorge X. Experiencias del personal de enfermería con pacientes hospitalizados por abuso de alcohol. Enferm. univ. 2017; 14(1): 19-27.

http://dx.doi.org/10.1016/j.reu.2016.12.001 
12. Falcó-Pegueroles A. Cuidar siguiendo los valores y principios éticos propios de la enfermería. Enferm. clín. 2005: 15(5): 287-90. http://dx.doi.org/10.1016/S1130-8621(05)71129-3

13. Morales-Castillo FA, Hernández-Cruz MC, Morales-Rodríguez MC, Landeros-Olvera EA. Validación y estandarización del instrumento: evaluación de los comportamientos de cuidado otorgado en enfermeras mexicanas. Enferm. univ. 2016; 13(1): 3-11. http://dx.doi.org/10.1016/j.reu.2015.11.005

14. Rodríguez S, Cárdenas M, Pacheco AL, Ramírez M, Ferro N, Alvarado E. Reflexión teórica sobre el arte del cuidado. Enferm. univ. 2017; 14(3): 191-8. http://dx.doi.org/10.1016/j.reu.2017.05.004

15. Mateo-Crisóstomo Y, Armendáriz-García NA, Alonso-Castillo MTJ, Martínez-Maldonado R. Conocimientos y creencias sobre el cuidado al usuario de alcohol por estudiantes de enfermería. Rev Cuid. 2016; 7(2): 1255-61. http://dx.doi.org/10.15649/cuidarte.v7i2.314

16. Amaro-Hinojosa MD, Campa-Magallón TJ, Cantú-Martínez PC, Gómez-Meza MV. Patrones de consumo de drogas licitas e ilícitas de los pacientes que ingresan a una sala de urgencias. Revenf. 2016; (30): 1-12. https://doi.org/10.15517/revenf.voi3o.22340

17. Lützén K, Dahlqvist V, Eriksson S, Norberg A. Developing the concept of moral sensitivity in health care practice. Nurs Ethics. 2006; 13(2): 187-96. http://dx.doi.org/10.1191/og69733006ne8370a

18. Lützén K, Blom T, Ewalds-Kvist B, Winch S.Moral stress, moral climate and moral sensitivity among psychiatric professionals.Nurs Ethics.2010;17(2):213-24.https://doi.org/10.1177/og69733009351951

19. Tuvesson $\mathrm{H}$, Lützen $\mathrm{K}$. Demographic factors associated with moral sensitivity among nursing students. Nurs Ethics. 2017; 24(7): 847-55. http://doi.org/10.1177/og69733015626602

20. Almaraz-Castruita DA, Alonso-Castillo BA, Alonso-Castillo MM, Olivia-Rodríguez NN, Alonso-Castillo MTJ. Sensibilidad ética en una población mexicana de enfermeras. Index Enferm. 2018; 27(1-2): 38-41. http://bitly.ws/dGKM

21. Calvo-Salguero A, Aguilar-Luzón MC, Salinas-Martínez de Lecea JM. Valores laborales individualistas y colectivistas de enfermeras y enfermeros en un entorno multicultural. Index Enferm. 2008; 17(4): 246-50. http://bitly.ws/dGLh

22. Almaraz-Castruita D, Alonso-Castillo B. Sensibilidad ética y su relación con el consumo de alcohol en el personal de enfermería. Rev Enferm Inst Mex Seguro Soc. 2016; 24(2): 123-8. http://bitly.ws/dGNo

23. Hernández-Ramírez G, Rivas-Acuña V, González-Suárez M, Victorino-Barra A. Relación de la sensibilidad ética y valores con el consumo de alcohol, tabaco y drogas médicas en docentes de enfermería de Tabasco, México. Cuba: XVI Coloquio Panamericano de Investigación en Enfermería; 2018. http://bitly.ws/dGNW

24. Alonso-Castillo MM, Alonso-Castillo BA, Armendariz-García NA, Rodríguez-Aguilar L, Esparza-Almanza SE. Sensibilidad ética, valores y estrés de conciencia en estudiantes profesores y profesionales de enfermería. Cuba: XVI Coloquio Panamericano de Investigación en Enfermería; 2018.

25. Kulju K, Suhonen R, Leino-Kilpi H. Ethical problems and moral sensitivity in physiotherapy: A descriptive study. Nurs Ethics. 2013; 20(5): 568-77. http://doi.org/10.1177/og69733012468462

26. Alonso CB, Alonso CM. Factores personales. Laborales, éticos, empatía e intuición como predictores de sensibilidad ética en estudiantes y profesionales de enfermería. Ensayos selectos en bioética. Capítulo II. México: Dem.; 2013: 24-36.

27. Ford R, Bammer G, Becker N. Improving nurses' therapeutic attitude to patients who use illicit drugs. Workplace drug and alcohol education is not enough. Int J Nurs Pract. 2009; 15(2): 112-8.

http://dx.doi.org/10.1111/j.1440-172X.2009.01732.x 